\title{
Moments and Estimation of the Exponentiated Moment Exponential Distribution
}

\author{
Devendra Kumar
}

(Communicated by T. ERBAY DALKILIC)

\begin{abstract}
A new extension of moment exponential distribution, called exponentiated moment exponential distribution $(E M E D)$, was recently introduced by Hasnain [14]. Based on lower generalized order statistics, we first derive the explicit expressions as well as recurrence relations for single and product moments of lower generalized order statistics and we use these results to compute the means, variances and coefficients of skewness and kurtosis of $E M E D$. Further, using a recurrence relation for single moment, we obtain characterization of $E M E D$. Next we obtain the maximum likelihood estimators of the unknown parameters and the approximate confidence intervals of the EMED. Finally, we consider Bayes estimation under the symmetric and asymmetric loss functions using gamma priors for both shape and scale parameters. We have are also derived the Bayes interval of this distribution. Monte Carlo simulations are performed to compare the performances of the proposed methods.
\end{abstract}

Keywords: explicit expression, Recurrence relation, Lower generalized order statistics, Order statistics, Record values, Exponentiated moment exponential distribution, Bayes estimator, General entropy loss function, Maximum likelihood estimator.

AMS Subject Classification (2010): Primary: 62G30,; Secondary: 62E10.

${ }^{*}$ Corresponding author

\section{Introduction}

The concept of generalized order statistics (gos) was introduced by Kamps [16] as a general framework for models of ordered random variables $\left(r v^{\prime} s\right)$. Moreover, many other models of ordered $r v^{\prime} s$, such as, ordinary order statistics (oos), order statistics with non-integral sample size (nonI), progressively type-II censored order statistics $(P C O)$, upper record values, upper Pfeifer records and sequential order statistics (sos) are seen to be particular cases of gos. These models can be effectively applied, e.g., in reliability theory. However, decreasingly ordered $r v^{\prime} s$ cannot be integrated into this framework. Consequently, this model is inappropriate to study, e.g. reversed ordered order statistic and lower record values models. Using the concept of gos Pawlas and Szynal [28] introduced the concept of lower generalized order statistics (lgos) to enable a common approach to descending order statistics, which was further studied by Burkschat et al. [6] with the name dual generalized order statistics. The lgos models enable us to study decreasingly ordered random variables like reversed order statistics, lower record values and lower Pfeifer records, through a common approach below:

Suppose $X^{*}(1, n, m, k), \ldots, X^{*}(n, n, m, k),(k \geq 1, m$ is a real number), are $n$ lgos from an absolutely continuous 
cumulative distribution function $(c d f) F(x)$ with probability density function $(p d f) f(x)$. Their joint $p d f$ of the form

$$
k\left(\prod_{j=1}^{n-1} \gamma_{j}\right)\left(\prod_{i=1}^{n-1}\left[F\left(x_{i}\right)\right]^{m} f\left(x_{i}\right)\right)\left[F\left(x_{n}\right)\right]^{k-1} f\left(x_{n}\right),
$$

for $F^{-1}(1)>x_{1} \geq x_{2} \geq \ldots \geq x_{n}>F^{-1}(0)$, where $\gamma_{j}=k+(n-j)(m+1)>0$ for all $j, 1 \leq j \leq n, k$ is a positive integer and $m \geq-1$. If $m=0$ and $k=1$, we obtain the joint pdf of the ordinary order statistics. If $k=1$ and $m=-1$, we obtain the joint $p d f$ of the first $n$ record values of the identically and independently distributed (iid) random variables with $c d f F(x)$ and corresponding $p d f f(x)$. Other statistics contained as particular cases include sos, progressively type II censored order statistics.

In view of (1.1), the marginal $p d f$ of the $r^{t h} \operatorname{lgos}, X^{*}(r, n, m, k) 1 \leq r \leq n$ is

$$
f_{X^{*}(r, n, m, k)}(x)=\frac{C_{r-1}}{(r-1) !}[F(x)]^{\gamma_{r}-1} f(x) g_{m}^{r-1}(F(x)) .
$$

The joint $p d f$ of $X^{*}(r, n, m, k)$ and $X^{*}(s, n, m, k), 1 \leq r<s \leq n$ is

$$
\begin{gathered}
f_{X^{*}(r, n, m, k), X^{*}(s, n, m, k)}(x, y)=\frac{C_{s-1}}{(r-1) !(s-r-1) !}[F(x)]^{m} f(x) g_{m}^{r-1}(F(x)) \\
\times\left[h_{m}(F(y))-h_{m}(F(x))\right]^{s-r-1}[F(y)]^{\gamma_{s}-1} f(y), \quad x>y,
\end{gathered}
$$

where $C_{r-1}=\prod_{i=1}^{r} \gamma_{i}, h_{m}(x)=-\ln x$, if $m=-1, h_{m}(x)=\frac{1}{m+1} x^{m+1}$, if $m \neq-1$ and $g_{m}(x)=h_{m}(x)-h_{m}(1), x \in$ $[0,1)$.

The recurrence relations for moments of ordered random variables are important because they reduce the amount of direct computations for moments, evaluation of higher moments and they can be used to characterize the distributions. The recurrence relations of higher joint moments enable us to derive single, product, triple and quadruple moments which can be used in Edgeworth approximate inference.

In the context of lgos, related topics have been studied by Pawlas and Szynal [28], Ahsanullah [1], Mbah and Ahsanullah [26] and Kumar [20, 21, 22]. Khan and Kumar [18] established recurrence relations for single and product moment of lgos from the exponentiated Pareto distribution. Keseling [17] characterized some continuous distributions based on conditional distributions of gos. Bieniek and Szynal [5] characterized some distributions via linearity of regression of gos. Cramer et al. [9] gave a unifying approach on characterization via linear regression of ordered random variables. The study of record values and associated statistics are of great significance in many real life situations such as meteorology, seismology, athletic events, economics, and life testing. The frequency of weather conditions inspired Chandler [8] to study the distributions of lower records, record times, and inter-record times for $i i d$ sequences of random variables. Computation of Fisher information for any particular distribution is quite important; see, for example, Zheng [35]. Fisher information matrix can be used to compute the asymptotic variances of the different functions of the estimators, for example, maximum likelihood estimators.

Recently, Hasnain [14] have introduced a new extension of moment exponential distribution, called exponentiated moment exponential distribution $(E M E D)$. He showed that this distribution is more flexible than Dara and Ahmad [10] moment exponential distribution and Gupta and Kundu [11] exponentiated exponential distribution. He obtained some basic properties and discussed unbiasedness and sufficiency of the parameters of EMED. He also discussed different properties of $E M E D$ of $n^{\text {th }}$ order statistics and finally obtained dual generalized order statistics for EMED. The moment distributions have applicability in numerous situations. For example, in the field of physics, Krumbein and Pettijohn [19] and Gy [13] studied moment distributions to examine the relation between particle diameter and its frequency. Preston [29] applied canonical distributions in ecology. Zelen [33] analyzed cell cycle and pulse labeling on length biased data. Zelen [34] also used length biased sample for the screening of disease and scheduling of examinations of patients. Brown [4] studied various aspects of the traffic streams. Warren [32] examined different case studies to observe the role of statistical distributions in forest and forestry products research. Taillie et al. [30] modelled populations of fish stocks using weighted distributions. Temkin [31] 
studied length biased survival models to analyze the cardiac transplantation situations and so on. In these and other application areas, it is pertinent that one has accurate estimation and prediction procedures based on records and moments of record values.

A random variable $X$ is said to have exponentiated moment exponential distribution $(E M E D)$ if its probability density function (pdf) is of the form

$$
f(x ; \alpha, \beta)=\frac{\alpha x}{\beta^{2}}\left[1-\left(1+\frac{x}{\beta}\right) e^{-x / \beta}\right]^{\alpha-1} e^{-x / \beta}, x>0, \alpha, \beta>0
$$

and the corresponding $c d f$ is

$$
F(x ; \alpha, \beta)=\left[1-\left(1+\frac{x}{\beta}\right) e^{-x / \beta}\right]^{\alpha}, x>0, \alpha, \beta>0,
$$

the survival function

$$
S(x ; \alpha, \beta)=1-\left[1-\left(1+\frac{x}{\beta}\right) e^{-x / \beta}\right]^{\alpha}, x>0, \alpha, \beta>0
$$

and the hazard function

$$
h(x ; \alpha, \beta)=\frac{\frac{\alpha x}{\beta^{2}}\left[1-\left(1+\frac{x}{\beta}\right) e^{-x / \beta}\right]^{\alpha-1} e^{-x / \beta}}{1-\left[1-\left(1+\frac{x}{\beta}\right) e^{-x / \beta}\right]^{\alpha}}, x>0, \alpha, \beta>0
$$

where the parameter $\alpha$ controls the shape of the distribution, while $\beta$ controls the scale of the distribution. EMED have a vital role in mathematics and statistics, in particular probability theory, in the perspective research related to ecology, reliability, biomedical field, econometric, survey sampling and in life testing. A recurrence relation for single and product moments of lgos from the EMED is obtained making use of the following differential equation (obtained from (1.4) and (1.5)).

$$
F(x ; \alpha, \beta)=\frac{\beta^{2}}{\alpha x}\left(e^{x / \beta}-1-\frac{x}{\beta}\right) f(x ; \alpha, \beta) .
$$
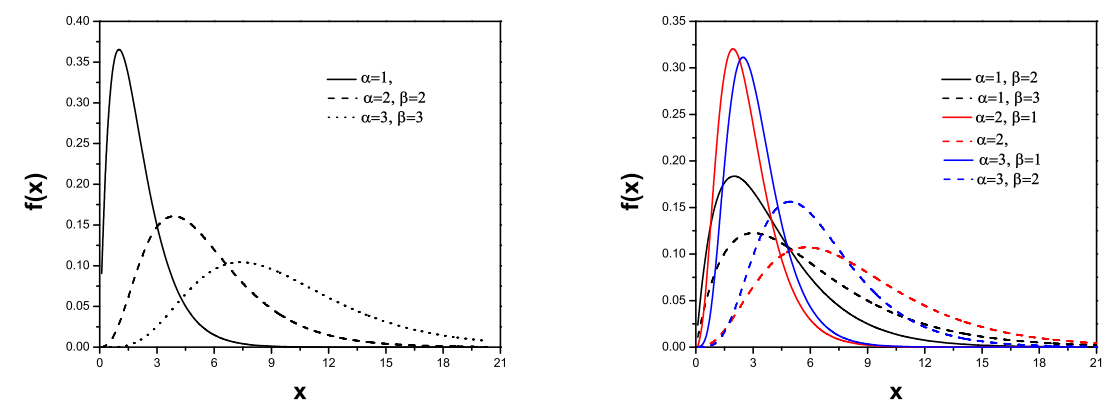

Figure 1. EME Density Function

Plotted above are the probability density function (figure 1), for $\alpha=1,2,3$ and $\beta=1,2,3$ and the hazard functions (figure 2) and Survival Function (figure 3) for $E M E D$ when $\alpha=1,2,3$ and $\beta=1,2,3$.

The presentation of the content of this work is as follows: In Section 2, we obtain explicit expressions and recurrence relations for single moments of lgos from $E M E D$. The explicit expressions and recurrence relations for product moments of lgos from EMED are discussed in Section 3. In Section 4, we characterize the distribution by using recurrence relation for single moment of $l g o s$. The Fisher information matrices of $E M E D$ are discussed in Section 5. We use maximum likelihood estimation $(M L E)$ method as a part of frequentist methodology for parameter estimation in Section 6. The asymptotic confidence intervals based on the observed Fisher's information matrix is also discussed here. Next, we consider Bayesian estimation of the unknown parameters in Section 7. 

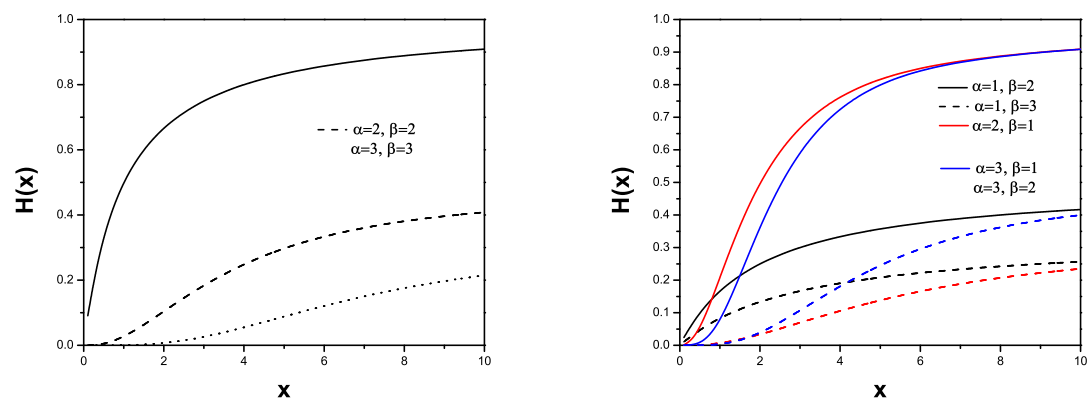

Figure 2. EME Hazard Function
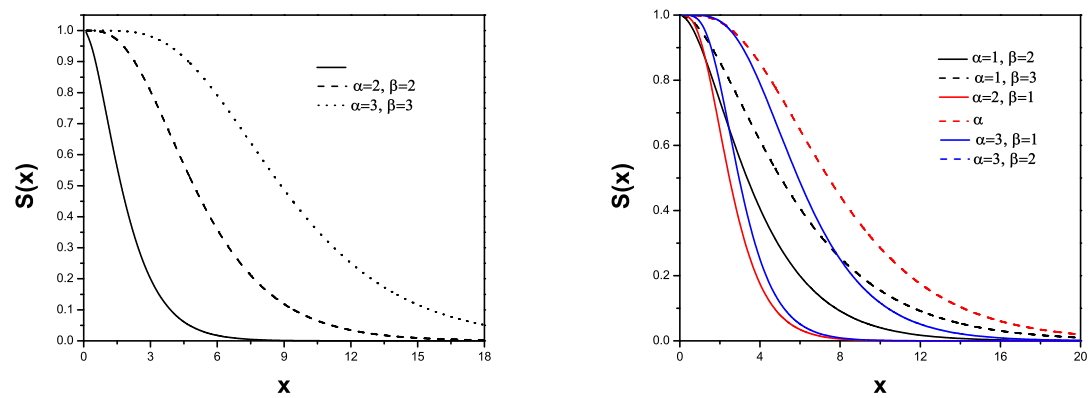

Figure 3. EME Survival Function

The Bayesian inference mainly depends on two features: choice of prior distribution of the parameters and the loss function to be used for Bayesian computations. In this article, we use gamma priors for both scale and shape parameters and they are assumed to be independent of each other. For Bayesian inference, we use a general entropy loss function. The main idea behind using this loss function is that with particular choices of the parameter involved in the form of loss function produces estimates under several well known loss functions, which are both symmetric and asymmetric in nature. A brief discussion of this loss function is presented later in this article in section 7 . The joint posterior distribution is complicated and thus the posterior sampling is not straightforward to implement. Here we propose a Markov Chain Monte Carlo technique which involve Metropolis-Hasting algorithm for posterior sampling. Besides Bayes estimates, we also obtain a two-sided Bayes probability intervals as a Bayesian counter part of the asymptotic confidence intervals in section 7. Bayes estimation heavily dependent on the choice of hyper parameters involved in the prior distributions, which is quite sensitive for the Bayesian inference. An alternative way to avoid the issue is to use an empirical Bayes estimation procedure for parameter estimation; section 8 introduces this procedure for the aforementioned distribution. The performances of the two estimation methods are compared by simulation in Section 9. In Section 10, two applications are demonstrated to illustrate the utility of the results derived in Section 3 and 4. Section 11 contains a brief conclusion.

\section{Relations for single moments}

For the EME distribution as given in (1.4), the $j$-th moments of $X^{*}(r, n, m, k)$ is given as

$$
\begin{aligned}
E\left[X^{* j}(r, n, m, k)\right] & =\int_{-\infty}^{\infty} x^{j} f_{X^{*}(r, n, m, k)}(x) d x \\
& =\frac{C_{r-1}}{(r-1) !} \int_{-\infty}^{\infty} x^{j}[F(x)]^{\gamma_{r}-1} f(x) g_{m}^{r-1}(F(x)) d x \\
& =\frac{C_{r-1}}{(r-1) !(m+1)^{r-1}} \sum_{u=0}^{r-1}(-1)^{u}\left(\begin{array}{c}
r-1 \\
u
\end{array}\right) \\
& \times \int_{-\infty}^{\infty} x^{j}[F(x)]^{\gamma_{r}-1} f(x) .
\end{aligned}
$$


Further, on using (1.4) and (1.5) in (2.1), and simplifying the resulting expression we get

$$
\begin{aligned}
E\left[X^{* j}(r, n, m, k)\right] & =\frac{\alpha C_{r-1}}{\beta^{j}(r-1) !(m+1)^{r-1}} \sum_{u=0}^{r-1} \sum_{p=0}^{\infty} \sum_{q=0}^{p}(-1)^{u+p}\left(\begin{array}{c}
r-1 \\
u
\end{array}\right) \\
& \times\left(\begin{array}{c}
\alpha \gamma_{r-u}-1 \\
p
\end{array}\right)\left(\begin{array}{c}
p \\
q
\end{array}\right) \frac{\Gamma(j+q+2)}{(p+1)^{j+p+2}} .
\end{aligned}
$$

and when $m=-1$ that

$$
\begin{gathered}
E\left[X^{* j}(r, n,-1, k)\right]=\frac{(\alpha k)^{r}}{\beta^{j}(r-1) !} \sum_{p=0}^{\infty} \sum_{u=0}^{\infty} \sum_{q=0}^{r-1+u+p}(-1)^{u} \alpha_{p}(r-1) \\
\times\left(\begin{array}{c}
\alpha k-1 \\
p
\end{array}\right)\left(\begin{array}{c}
r-1+u+p \\
q
\end{array}\right) \frac{\Gamma(j+q+2)}{(r+p+u)^{j+p+2}} .
\end{gathered}
$$

where $\alpha_{p}(r-1)$ is the coefficient of $\left[\left(1+\frac{x}{\beta}\right) e^{-x / \beta}\right]^{r-1+p}$ in the expansion of $\left[\sum_{u=1}^{\infty} \frac{1}{u}\left(1+\frac{x}{\beta}\right) e^{-x / \beta}\right]^{r-1}$ (see Balakrishnan and Cohan [3]).

If $\alpha$ is a positive integer, the relations (2.2) and (2.3) then give

$$
\begin{aligned}
E\left[X^{* j}(r, n, m, k)\right]= & \frac{\alpha C_{r-1}}{\beta^{j}(r-1) !(m+1)^{r-1}} \sum_{u=0}^{r-1} \sum_{p=0}^{\alpha \gamma_{r-u}-1} \sum_{q=0}^{p}(-1)^{u+p}\left(\begin{array}{c}
r-1 \\
u
\end{array}\right) \\
& \times\left(\begin{array}{c}
\alpha \gamma_{r-u}-1 \\
p
\end{array}\right)\left(\begin{array}{c}
p \\
q
\end{array}\right) \frac{\Gamma(j+q+2)}{(p+1)^{j+p+2}} .
\end{aligned}
$$

and

$$
\begin{aligned}
E\left[X^{* j}(r, n,-1, k)\right] & =\frac{(\alpha k)^{r}}{\beta^{j}(r-1) !} \sum_{p=0}^{\infty} \sum_{u=0}^{\alpha k-1} \sum_{q=0}^{r-1+u+p}(-1)^{u} \alpha_{p}(r-1)\left(\begin{array}{c}
\alpha k-1 \\
p
\end{array}\right) \\
& \times\left(\begin{array}{c}
r-1+u+p \\
q
\end{array}\right) \frac{\Gamma(j+q+2)}{(r+p+u)^{j+p+2}} .
\end{aligned}
$$

\section{Special cases}

i) Putting $m=0, k=1$ in (2.4), the explicit formula for the single moments of ordinary order statistics of the $E M E D$ can be obtained as

$$
\begin{aligned}
E\left[X_{n-r+1: n}^{j}\right] & =\frac{\alpha C_{r: n}}{\beta^{j}} \sum_{u=0}^{r-1} \sum_{p=0}^{\alpha(n-r+1)-1} \sum_{q=0}^{p}(-1)^{u+p}\left(\begin{array}{c}
r-1 \\
u
\end{array}\right) \\
& \times\left(\begin{array}{c}
\alpha(n-r+1)-1 \\
p
\end{array}\right)\left(\begin{array}{c}
p \\
q
\end{array}\right) \frac{\Gamma(j+q+2)}{(p+1)^{j+p+2}} .
\end{aligned}
$$

That is

$$
\begin{aligned}
E\left[X_{r: n}^{j}\right] & =\frac{\alpha C_{r: n}}{\beta^{j}} \sum_{u=0}^{n-r} \sum_{p=0}^{\alpha r-1} \sum_{q=0}^{p}(-1)^{u+p}\left(\begin{array}{c}
n-r \\
u
\end{array}\right)\left(\begin{array}{c}
\alpha r-1 \\
p
\end{array}\right) \\
& \times\left(\begin{array}{c}
p \\
q
\end{array}\right) \frac{\Gamma(j+q+2)}{(p+1)^{j+p+2}},
\end{aligned}
$$

where

$$
C_{r: n}=\frac{n !}{(r-1) !(n-r) !}
$$


ii) Putting $k=1$ in (2.5), the explicit expressions for the moments of lower record values for the EMED can be obtained as

$$
\begin{aligned}
E\left[X_{L(r)}^{j}\right] & =\frac{\alpha^{r}}{\beta^{j}(r-1) !} \sum_{p=0}^{\infty} \sum_{u=0}^{\alpha k-1} \sum_{q=0}^{r-1+u+p}(-1)^{u} \alpha_{p}(r-1) \\
& \times\left(\begin{array}{c}
\alpha-1 \\
p
\end{array}\right)\left(\begin{array}{c}
r-1+u+p \\
q
\end{array}\right) \frac{\Gamma(j+q+2)}{(r+p+u)^{j+p+2}} .
\end{aligned}
$$

A recurrence relation for moments of lgos from (1.5) can be obtained in the following theorem.

Theorem 2.1. For the distribution as given in (1.5) and for $2 \leq r \leq n, n \geq 2$ and $k=1,2, \ldots$

$$
\begin{aligned}
E\left[X^{* j}(r, n, m, k)\right] & =E\left[X^{* j}(r-1, n, m, k)\right]=\frac{j \beta^{2}}{\alpha \gamma_{r}}\left\{E\left[X^{* j-2}(r, n, m, k)\right]\right. \\
& \left.+\frac{1}{\beta} E\left[X^{* j-1}(r, n, m, k)\right]-E\left[\phi\left(X^{*}(r, n, m, k)\right)\right]\right\}
\end{aligned}
$$

where

$$
\phi(x)=x^{j-2} e^{x / \beta}
$$

Proof: From (1.2), we have

$$
E\left[X^{* j}(r, n, m, k)\right]=\frac{C_{r-1}}{(r-1) !} \int_{0}^{\infty} x^{j}[F(x)]^{\gamma_{r}-1} f(x) g_{m}^{r-1}(F(x)) d x .
$$

By integrating by parts, we get

$$
\begin{aligned}
E\left[X^{* j}(r, n, m, k)\right] & -E\left[X^{* j}(r-1, n, m, k)\right] \\
& =-\frac{j C_{r-1}}{\gamma_{r}(r-1) !} \int_{0}^{\infty} x^{j-1}[F(x)]^{\gamma_{r}} g_{m}^{r-1}(F(x)) d x \\
& =-\frac{j C_{r-1}}{\gamma_{r}(r-1) !} \int_{0}^{\infty} x^{j-1}[F(x)]^{\gamma_{r}-1} f(x) \\
& \times\left\{\frac{\beta^{2}}{\alpha x}\left(e^{x / \beta}-\frac{x}{\beta}-1\right)\right\} g_{m}^{r-1}(F(x)) d x . \\
& =-\frac{j \beta^{2} C_{r-1}}{\alpha \gamma_{r}(r-1) !} \int_{0}^{\infty} x^{j-2} e^{x / \beta}[F(x)]^{\gamma_{r}-1} f(x) g_{m}^{r-1}(F(x)) d x \\
& +\frac{j \beta^{2} C_{r-1}}{\alpha \gamma_{r}(r-1) !} \int_{0}^{\infty} x^{j-2}[F(x)]^{\gamma_{r}-1} f(x) g_{m}^{r-1}(F(x)) d x \\
& +\frac{j \beta C_{r-1}}{\alpha \gamma_{r}(r-1) !} \int_{0}^{\infty} x^{j-1}[F(x)]^{\gamma_{r}-1} f(x) g_{m}^{r-1}(F(x)) d x .
\end{aligned}
$$

The result follows.

Remark 2.1. Under the assumptions of Theorem 2.1, with $k=1 ; m=0$, we shall deduce the recurrence relation for single moments of ordinary order statistics from EMED.

Remark 2.2. Putting $k=0 ; m=-1$ in Theorem 2.1, we obtain the recurrence relation for single moments of lower record values from $E M E D$.

\section{Relations for product moments}

For $E M E D$, the product moments of $X^{*}(r, n, m, k)$ and $X^{*}(s, n, m, k)$ is given as

$$
E\left[X^{* i}(r, n, m, k) X^{* j}(s, n, m, k)\right]=\int_{-\infty}^{\infty} \int_{y}^{\infty} x^{i} y^{j} f_{X^{*}(r, n, m, k) X^{*}(s, n, m, k)}(x, y) d x d y .
$$




$$
\begin{gathered}
=\frac{C_{s-1}}{(r-1) !(s-r-1) !(m+1)^{s-2}} \sum_{a=0}^{r-1} \sum_{b=0}^{s-r-1}(-1)^{u+v}\left(\begin{array}{c}
r-1 \\
u
\end{array}\right)\left(\begin{array}{c}
s-r-1 \\
v
\end{array}\right) \\
\times \int_{0}^{\infty} y^{j}[F(y)]^{\gamma_{s-b}-1} f(y) G(y) d y, x>y .
\end{gathered}
$$

where

$$
\begin{gathered}
G(y)=\int_{y}^{\infty} x^{i}[F(y)]^{(s-r+a-b)(m+1)-1} f(x) d x \\
=\alpha \beta^{i} \sum_{c=0}^{\infty} \sum_{d=0}^{c} \sum_{p=0}^{i+d+1}(-1)^{c}\left(\begin{array}{c}
\alpha(s-r+a-b)(m+1)-1 \\
c
\end{array}\right) \\
\times\left(\begin{array}{c}
c \\
d
\end{array}\right) \frac{e^{-[(c+1) / \beta] y}[((c+1) / \beta) y]^{p} \Gamma(i+d+2)}{p !(c+1)^{i+d+2}} .
\end{gathered}
$$

On substituting the above expression of $G(y)$ in (3.1), and simplifying the resulting expression we get

$$
\begin{gathered}
E\left[X^{* i}(r, n, m, k) X^{* j}(s, n, m, k)\right]=\frac{\alpha^{2} \beta^{i+j} C_{s-1}}{(r-1) !(s-r-1) !(m+1)^{s-2}} \sum_{a=0}^{r-1} \sum_{b=0}^{s-r-1} \\
\times \sum_{c=0}^{\infty} \sum_{d=0}^{c} \sum_{p=0}^{i+d+1} \sum_{u=0}^{\infty} \sum_{v=0}^{u}(-1)^{a+b+c+u}\left(\begin{array}{c}
r-1 \\
a
\end{array}\right)\left(\begin{array}{c}
s-r-1 \\
b
\end{array}\right) \\
\times\left(\begin{array}{c}
\alpha(s-r+a-b)(m+1)-1 \\
c
\end{array}\right)\left(\begin{array}{c}
c \\
d
\end{array}\right)\left(\begin{array}{c}
\alpha \gamma_{s-b}-1 \\
u
\end{array}\right)\left(\begin{array}{l}
u \\
v
\end{array}\right) \\
\times \frac{\Gamma(i+d+2) \Gamma(j+p+v+2)}{p !(c+1)^{i+d+2-p}(c+u+2)^{j+p+v+2}} .
\end{gathered}
$$

and when $m=-1$ that

$$
\begin{gathered}
E\left[X^{* i}(r, n,-1, k) X^{* j}(s, n,-1, k)\right]=\frac{\beta^{i+j}(\alpha k)^{s}}{(r-1) !(s-r-1) !} \sum_{a=0}^{s-r-1} \sum_{b=0}^{\infty} \sum_{c=0}^{s-a-2+p+b} \sum_{d=0}^{i+c+1} \\
\times \sum_{p=0}^{\infty} \sum_{q=0}^{\infty} \sum_{u=0}^{\infty} \sum_{v=0}^{u+a+q}(-1)^{s-r-1+a+u}\left(\begin{array}{c}
s-r-1 \\
a
\end{array}\right)\left(\begin{array}{c}
s-a-2+b+p \\
c
\end{array}\right)\left(\begin{array}{c}
\alpha k-1 \\
u
\end{array}\right) \\
\times\left(\begin{array}{c}
u+a+q \\
v
\end{array}\right) \frac{\Gamma(i+c+2) \Gamma(j+d+v+2)}{d !(s-a-1+b+p)^{i+c+2-d}(s+b+p+q+u)^{j+d+v+2}} .
\end{gathered}
$$

If $\alpha$ is a positive integer, then the relations (3.2) and (3.3) take the form

$$
\begin{gathered}
E\left[X^{* i}(r, n, m, k) X^{* j}(s, n, m, k)\right]=\frac{\alpha^{2} \beta^{i+j} C_{s-1}}{(r-1) !(s-r-1) !(m+1)^{s-2}} \sum_{a=0}^{r-1} \sum_{b=0}^{s-r-1} \\
\times \sum_{c=0}^{\alpha(s-r+a-b)(m+1)-1} \sum_{d=0}^{c} \sum_{p=0}^{i+d+1} \sum_{u=0}^{\alpha \gamma_{s-b}-1} \sum_{v=0}^{u}(-1)^{a+b+c+u}\left(\begin{array}{c}
r-1 \\
a
\end{array}\right)\left(\begin{array}{c}
s-r-1 \\
b
\end{array}\right) \\
\times\left(\begin{array}{c}
\alpha(s-r+a-b)(m+1)-1 \\
c
\end{array}\right)\left(\begin{array}{c}
c \\
d
\end{array}\right)\left(\begin{array}{c}
\alpha \gamma_{s-b}-1 \\
u
\end{array}\right)\left(\begin{array}{c}
u \\
v
\end{array}\right) \\
\times \frac{\Gamma(i+d+2) \Gamma(j+p+v+2)}{p !(c+1)^{i+d+2-p}(c+u+2)^{j+p+v+2}} .
\end{gathered}
$$

and

$$
E\left[X^{* i}(r, n,-1, k) X^{* j}(s, n,-1, k)\right]=\frac{\beta^{i+j}(\alpha k)^{s}}{(r-1) !(s-r-1) !} \sum_{a=0}^{s-r-1} \sum_{b=0}^{\infty} \sum_{c=0}^{s-a-2+p+b} \sum_{d=0}^{i+c+1}
$$




$$
\begin{gathered}
\times \sum_{p=0}^{\infty} \sum_{q=0}^{\infty} \sum_{u=0}^{\alpha k-1} \sum_{v=0}^{u+a+q}(-1)^{s-r-1+a+u}\left(\begin{array}{c}
s-r-1 \\
a
\end{array}\right)\left(\begin{array}{c}
s-a-2+b+p \\
c
\end{array}\right)\left(\begin{array}{c}
\alpha k-1 \\
u
\end{array}\right) \\
\quad \times\left(\begin{array}{c}
u+a+q \\
v
\end{array}\right) \frac{\Gamma(i+c+2) \Gamma(j+d+v+2)}{d !(s-a-1+b+p)^{i+c+2-d}(s+b+p+q+u)^{j+d+v+2}} .
\end{gathered}
$$

\section{Special cases}

i) Putting $m=0, k=1$ in (3.4), we obtain the explicit formula for the product moments of ordinary order statistics for the $E M E D$.

ii) Putting $k=1$ in (3.5), we obtain the explicit expressions for the product moments of lower record values for the EMED.

Making use of (1.6), we can derive the recurrence relations for product moments of lgos.

Theorem 3.1. For the distribution given in (1.5) and for $1 \leq r<s \leq n, n \geq 2$ and $k=1,2, \ldots$

$$
\begin{gathered}
E\left[X^{* i}(r, n, m, k) X^{* j}(s, n, m, k)\right]-E\left[X^{* i}(r, n, m, k) X^{* j}(s-1, n, m, k)\right] \\
+\frac{\beta^{2} j}{\alpha \gamma_{s}}\left\{E\left[X^{* i}(r, n, m, k) X^{* j-2}(s, n, m, k)\right]+\frac{1}{\beta} E\left[X^{* i}(r, n, m, k) X^{* j-1}(s, n, m, k)\right]\right\} \\
-\frac{\beta^{2} j}{\alpha \gamma_{s}} E\left[\phi\left(X^{*}(r, n, m, k) X^{*}(s, n, m, k)\right)\right],
\end{gathered}
$$

where

$$
\phi(x, y)=x^{i} y^{j-2} e^{y / \beta}
$$

Proof: From (1.3), we have

$$
\begin{aligned}
E\left[X^{* i}(r, n, m, k) X^{* j}(s, n, m, k)\right] & =\frac{C_{s-1}}{(r-1) !(s-r-1) !} \int_{0}^{\infty} \int_{0}^{x} x^{i} y^{j}[F(x)]^{m} f(x) \\
& \times g_{m}^{r-1}(F(x))\left[h_{m}(F(y))-h_{m}(F(x))\right]^{s-r-1} \\
& \times[F(y)]^{\gamma_{s}-1} f(y) d y d x .
\end{aligned}
$$

By integrating by parts we get

$$
\begin{aligned}
& E\left[X^{* i}(r, n, m, k) X^{* j}(s, n, m, k)\right]-E\left[X^{* i}(r, n, m, k) X^{* j-1}(s-1, n, m, k)\right] \\
& \quad=\frac{j C_{s-1}}{\gamma_{s}(r-1) !(s-r-1) !} \int_{0}^{\infty} \int_{0}^{x} x^{i} y^{j-1}[F(x)]^{m} f(x) g_{m}^{r-1}(F(x)) \\
& \quad \times\left[h_{m}(F(y))-h_{m}(F(x))\right]^{s-r-1}[F(y)]^{\gamma_{s}-1} f(y) d y d x
\end{aligned}
$$

On using the relation (1.8) in (3.7), we get the result given in (3.6).

Remark 3.1. Under the assumptions of Theorem 3.1, with $k=1 ; m=0$, we shall deduce the recurrence relations for product moments of ordinary order statistics from EMED.

Remark 3.2. Putting $k=0 ; m=-1$ in Theorem 3.1, we obtain the recurrence relations for product moments of lower record values from $E M E D$.

Remark 3.3. At $i=0$, Theorem 3.1 reduces to Theorem 2.1. 


\section{Characterization}

In this section, we shall characterize $E M E D$ based on recurrence relation for single moment of $l g o s$. Let $L(a, b)$ stand for the space of all integrable functions on $(a, b)$. A sequence $\left(f_{n}\right) \subset L(a, b)$ is called complete on $L(a, b)$ if for all functions $g \in L(a, b)$ the condition

$$
\int_{a}^{b} g(x) f_{n}(x) d x=0, \quad n \in N
$$

implies $g(x)=0$ a.e. on $(a, b)$. We start with the following result of Lin [24].

Proposition 1: Let $n_{0}$ be any fixed non-negative integer, $-\infty \leq a<b \leq \infty$ and $g(x) \geq 0$ an absolutely continuous function with $g^{\prime}(x) \neq 0$ a.e. on $(a, b)$. Then the sequence of functions $\left\{(g(x))^{n} e^{-g(x)}, n \geq n_{0}\right\}$ is complete in $L(a, b)$ iff $g(x)$ is strictly monotone on $(a, b)$.

Using the above Proposition we get a stronger version of Theorem 2.1.

Theorem 4.1. Let $X$ be a non-negative random variable having an absolutely continuous distribution function $F(x)$ with $F(0)=0$ and $0<F(x)<1$ for all $x>0$, then

$$
\begin{aligned}
E\left[X^{* j}(r, n, m, k)\right] & =E\left[X^{* j}(r-1, n, m, k)\right]=\frac{j \beta^{2}}{\alpha \gamma_{r}}\left\{E\left[X^{* j-2}(r, n, m, k)\right]\right. \\
& \left.+\frac{1}{\beta} E\left[X^{* j-1}(r, n, m, k)\right]-E\left[\phi\left(X^{*}(r, n, m, k)\right)\right]\right\} .
\end{aligned}
$$

if and only if

$$
F(x ; \alpha, \beta)=\left[1-\left(1+\frac{x}{\beta}\right) e^{-x / \beta}\right]^{\alpha}, x>0, \alpha, \beta>0 .
$$

Proof. The necessary part follows immediately from equation (2.6). On the other hand if the recurrence relation in equation (4.1) is satisfied, then on using equation (1.2), we have

$$
\begin{aligned}
& \frac{C_{r-1}}{(r-1) !} \int_{0}^{\infty} x^{j}[F(x)]^{\gamma_{r}-1} f(x) g_{m}^{r-1}(F(x)) d x \\
= & \frac{(r-1) C_{r-1}}{\gamma_{r}(r-1) !} \int_{0}^{\infty} x^{j}[F(x)]^{\gamma_{r}+m} f(x) g_{m}^{r-2}(F(x)) d x \\
- & \frac{\beta^{2} j C_{r-1}}{\alpha \gamma_{r}(r-1) !} \int_{0}^{\infty} x^{j-2} e^{x / \beta}[F(x)]^{\gamma_{r}-1} f(x) g_{m}^{r-1}(F(x)) d x \\
+ & \frac{\beta^{2} j C_{r-1}}{\alpha \gamma_{r}(r-1) !} \int_{0}^{\infty} x^{j-2}[F(x)]^{\gamma_{r}-1} f(x) g_{m}^{r-1}(F(x)) d x \\
+ & \frac{\beta j C_{r-1}}{\alpha \gamma_{r}(r-1) !} \int_{0}^{\infty} x^{j-1}[F(x)]^{\gamma_{r}-1} f(x) g_{m}^{r-1}(F(x)) d x .
\end{aligned}
$$

Integrating the first integral on the right hand side of equation (4.2), by parts, we get

$$
\begin{gathered}
\frac{j C_{r-1}}{\gamma_{r}(r-1) !} \int_{0}^{\infty} x^{j-1}[F(x)]^{\gamma_{r}-1} f(x) g_{m}^{r-1}(F(x)) d x \\
\times\left\{F(x)-\frac{\beta^{2}}{\alpha x e^{-x / \beta}} f(x)+\frac{\beta^{2}}{\alpha x} f(x)+\frac{\beta}{\alpha} f(x)\right\} d x=0 .
\end{gathered}
$$

It now follows from Proposition 1

$$
F(x ; \alpha, \beta)=\frac{\beta^{2}}{\alpha x}\left(e^{x / \beta}-1-\frac{x}{\beta}\right) f(x ; \alpha, \beta),
$$

which proves that

$$
F(x ; \alpha, \beta)=\left[1-\left(1+\frac{x}{\beta}\right) e^{-x / \beta}\right]^{\alpha}, x>0, \alpha, \beta>0 .
$$

Remark 4.1. For $k=1, m=0$ and $k=1, m=-1$, we obtain the characterization results of the $E M E D$ based on order statistics and record values, respectively. 


\section{Fisher Information Matrix}

The Fisher information matrix summarizes the amount of information in the data relative to the quantities of interest. There are many applications of the information matrix in modeling, systems analysis, and estimation, including confidence region calculation, input design, prediction bounds, and "noninformative" priors for Bayesian analysis. Let $X$ be a continuous random variable with $c d f F(x ; \Theta)$ and the $p d f f(x ; \Theta)$. For the simplicity, we consider only two parameters $\alpha$ and $\beta$, although the results are true for any finite-dimensional vector. Under the standard regularity conditions Gupta and Kundu [12], the Fisher information matrix for the parameter vector $\Theta=(\alpha, \beta)$ based on an observation in terms of the expected values of the first and second derivatives of the log-likelihood function is

$$
\begin{aligned}
I(\Theta) & =\frac{1}{n}\left(\begin{array}{ll}
E\left(-\frac{\partial^{2} \ln L(\alpha, \beta)}{\partial^{2} \alpha}\right) & E\left(-\frac{\partial^{2} \ln L(\alpha, \beta)}{\partial \alpha \partial \beta}\right) \\
E\left(-\frac{\partial^{2} \ln L(\alpha, \beta)}{\partial \alpha \partial \beta}\right) & E\left(-\frac{\partial^{2} \ln L(\alpha, \beta)}{\partial^{2} \beta}\right)
\end{array}\right) \\
& =\frac{1}{n}\left(\begin{array}{ll}
\phi_{11} & \phi_{12} \\
\phi_{21} & \phi_{22}
\end{array}\right),
\end{aligned}
$$

where

$$
\phi_{i j}=\int_{-\infty}^{\infty}\left(\frac{\partial}{\partial \Theta_{i}} f(x ; \Theta)\right)\left(\frac{\partial}{\partial \Theta_{j}} f(x ; \Theta)\right) f(x ; \Theta) d x \text { for } i, j=1,2 .
$$

Now, we will derive Fisher information matrix of EMED. It can be shown that

$$
\ln f(x ; \alpha, \beta)=\ln \alpha+\ln x-2 \ln \beta-\frac{x}{\beta}+(\alpha-1) \ln \left[1-\left(1+\frac{x}{\beta}\right) e^{-x / \beta}\right] .
$$

Taking partial derivatives with respect to $\alpha$ and $\beta$ respectively from (5.3), we have

$$
\frac{\partial \ln f(x ; \alpha, \beta)}{\partial \alpha}=\frac{1}{\alpha}+\ln \left[1-\left(1+\frac{x}{\beta}\right) e^{-x / \beta}\right]
$$

and

$$
\frac{\partial \ln f(x ; \alpha, \beta)}{\partial \beta}=-\frac{2}{\beta}+\frac{x}{\beta^{2}}-\frac{x^{2} e^{-x / \beta}(\alpha-1)}{\beta^{3}\left[1-\left(1+\frac{x}{\beta}\right) e^{-x / \beta}\right]} .
$$

Therefore, the second partial derivatives are

$$
\begin{gathered}
\frac{\partial^{2} \ln f(x ; \alpha, \beta)}{\partial \alpha^{2}}=-\frac{1}{\alpha^{2}} \\
\frac{\partial^{2} \ln f(x ; \alpha, \beta)}{\partial \beta^{2}}=\frac{2}{\beta^{2}}-\frac{2 x}{\beta^{3}}+\frac{x^{2} e^{-x / \beta}(\alpha-1)}{\beta^{4}\left[1-\left(1+\frac{x}{\beta}\right) e^{-x / \beta}\right]} \\
-\frac{x^{3} e^{-x / \beta}(\alpha-1)}{\beta^{5}\left[1-\left(1+\frac{x}{\beta}\right) e^{-x / \beta}\right]}-\frac{x^{3} e^{-2 x / \beta}(\alpha-1)\left[1-\left(1+\frac{x}{\beta}\right)\right]}{\beta^{5}\left[1-\left(1+\frac{x}{\beta}\right) e^{-x / \beta}\right]^{2}}
\end{gathered}
$$

and

$$
\frac{\partial^{2} \ln f(x ; \alpha, \beta)}{\partial \alpha \partial \beta}=-\frac{x^{2} e^{-x / \beta}}{\beta^{4}\left[1-\left(1+\frac{x}{\beta}\right) e^{-x / \beta}\right]} .
$$

Thus the elements of Fisher information matrix for single observation from $E M E D$ is in the form

$$
\phi_{11}=E\left(-\frac{\partial^{2} \ln L(\alpha, \beta)}{\partial^{2} \alpha}\right)=\frac{1}{\alpha},
$$




$$
\phi_{12}=\phi_{21}=E\left(-\frac{\partial^{2} \ln L(\alpha, \beta)}{\partial \alpha \partial \beta}\right)=\frac{\beta\left[2-\alpha\left\{2+\psi_{1}(\alpha)\right\}\right]}{\alpha-1}
$$

and

$$
\phi_{22}=E\left(-\frac{\partial^{2} \ln L(\alpha, \beta)}{\partial^{2} \beta}\right)=\frac{\alpha^{2} \beta^{2}\left[\psi_{2}(\alpha)-\left(4+(2 / \alpha) \psi_{1}(\alpha)-2\right]\right.}{\alpha-1},
$$

where

$$
\psi_{j}(\alpha)=\sum_{u=0}^{\infty} \sum_{v=0}^{u}(-1)^{u}\left(\begin{array}{c}
\alpha-1 \\
u
\end{array}\right)\left(\begin{array}{c}
u \\
v
\end{array}\right) \frac{\Gamma(j+v+2)}{(1+u)^{j+v+2}}, j=1,2, \ldots
$$

Moreover, Fisher information matrix for a sample of size $n$ from $E M E$ distribution is simple $n I(\alpha, \beta)$.

\section{Non-Bayesian estimation}

In this section we discuss the process of obtaining the maximum likelihood estimators of the parameters $\alpha$ and $\beta$ of the $E M E D$. Suppose $X_{1}, X_{2}, \ldots, X_{n}$ be the random sample with observed values $x_{1}, x_{2}, \ldots, x_{n}$ from $E M E D$. Let $\Theta=(\alpha, \beta)$ be the parameter vector. The likelihood function based on the observed random sample of size $n$ is obtained from

$$
L(\alpha, \beta ; x)=\frac{\alpha^{n}}{\beta^{2 n}} \prod_{i=1}^{n}\left[1-\left(1+\frac{x_{i}}{\beta}\right) e^{-x_{i} / \beta}\right]^{\alpha-1} x_{i} e^{-x_{i} / \beta} .
$$

The maximum likelihood estimates are the values of $\alpha$ and $\beta$ that maximize this likelihood function.

\subsection{Maximum likelihood estimation}

The $\log$ likelihood function $l(\alpha, \beta \mid x)=\log L(\alpha, \beta \mid x)$, dropping terms that do not involve $\alpha$ and $\beta$, is

$$
\begin{aligned}
l(\alpha, \beta \mid x) & =n(\ln \alpha-2 \ln \beta)+\sum_{i=1}^{n} \ln x_{i}-\frac{1}{\beta} \sum_{i=1}^{n} x_{i} \\
& +(\alpha-1) \sum_{i=1}^{n} \ln \left[1-\left(1+\frac{x_{i}}{\beta}\right) e^{-x_{i} / \beta}\right] .
\end{aligned}
$$

We assume that the parameters $\alpha$ and $\beta$ are unknown. To obtain the normal equations for the unknown parameters, we differentiate (6.2) partially with respect to $\alpha$ and $\beta$ and equate to zero. The resulting equations are

$$
\begin{gathered}
0=\frac{\partial l(\alpha, \beta \mid x)}{\partial \alpha}=\frac{n}{\alpha}+\sum_{i=1}^{n} \ln \left[1-\left(1+\frac{x_{i}}{\beta}\right) e^{-x_{i} / \beta}\right] \\
0=\frac{\partial l(\alpha, \beta \mid x)}{\partial \beta}=-\frac{2 n}{\beta}+\frac{1}{\beta^{2}} \sum_{i=1}^{n} x_{i}-\sum_{i=1}^{n} \frac{(\alpha-1) x_{i}^{2} e^{-x_{i} / \beta}}{\beta^{3}\left[1-\left(1+\frac{x_{i}}{\beta}\right) e^{-x_{i} / \beta}\right]} .
\end{gathered}
$$

The solutions of the above equations are the maximum likelihood estimators of the EMED parameters $\alpha$ and $\beta$, denoted $\hat{\alpha}_{M L E}$ and $\hat{\beta}_{M L E}$, respectively. As the equations expressed in (6.3) and (6.4) cannot be solved analytically, one must use a numerical procedure to solve them.

The profile log-likelihood of $\beta$ can be obtained by Substituting $\hat{\alpha}_{M L E}$ from (6.3) in (6.2),

$$
\begin{aligned}
h(\beta) & =\ln L[\hat{\alpha}(\beta), \beta]=n \ln n-n \ln \left\{\sum_{i=1}^{n} \ln \left[1-\left(1+\frac{x_{i}}{\beta}\right) e^{-x_{i} / \beta}\right]\right\} \\
& -n+2 n \ln \beta-\frac{1}{\beta} \sum_{i=1}^{n} x_{i}-\sum_{i=1}^{n} \ln \left[1-\left(1+\frac{x_{i}}{\beta}\right) e^{-x_{i} / \beta}\right] .
\end{aligned}
$$

Therefore, the $M L E$ of $\beta$, say $\hat{\beta}_{M L E}$ can be obtained by minimizing (6.5) with respect to $\beta$ as follows:

$$
\frac{\partial h(\beta)}{\partial \beta}=\sum_{i=1}^{n} \frac{x_{i}^{2} e^{-x_{i} / \beta}}{\beta^{3}\left[1-\left(1+\frac{x_{i}}{\beta}\right) e^{-x_{i} / \beta}\right]}+\frac{2 n}{\beta}+\frac{1}{\beta^{2}} \sum_{i=1}^{n} x_{i}
$$




$$
-\frac{n}{\sum_{i=1}^{n} \ln \left[1-\left(1+\frac{x_{i}}{\beta}\right) e^{-x_{i} / \beta}\right]} \sum_{i=1}^{n} \frac{x_{i}^{2} e^{-x_{i} / \beta}}{\beta^{3}\left[1-\left(1+\frac{x_{i}}{\beta}\right) e^{-x_{i} / \beta}\right]} .
$$

Once $\hat{\beta}_{M L E}$ is obtained, the $M L E$ of $\alpha$ say $\hat{\alpha}_{M L E}$ can be obtained from (6.4) as $\hat{\alpha}_{M L E}=\hat{\alpha}\left(\beta_{M L E}\right)$.

Now, consider the $M L E$ of $\alpha$ when the scale parameter $\beta$ is known. Without loss of generality, we can take $\beta=1$. If $\beta$ is known, the $M L E$ of $\alpha$ say $\hat{\alpha}_{M L E S C K}$, is

$$
\hat{\alpha}_{M L E S C K}=-\frac{n}{\sum_{i=1}^{n} \ln \left[1-\left(1+\frac{x_{i}}{\beta}\right) e^{-x_{i} / \beta}\right]} .
$$

It follows, by the asymptotic properties of the $M L E$, that

$$
\hat{\alpha}_{M L E S C K} \approx N\left(\alpha, \frac{1}{n \phi_{11}}\right)
$$

where $\phi_{11}$ is the single information about $\alpha$ which is define in (5.4).

Let us consider the $M L E$ of $\beta$ when the shape parameter $\alpha$ is known. For known $\alpha$ the $M L E$ of $\beta$ say $\hat{\beta}_{M L E S H K}$ can be obtained by numerical solving of the following equation:

$$
\frac{1}{\beta^{2}} \sum_{i=1}^{n} x_{i}-\frac{2 n}{\beta}-\sum_{i=1}^{n} \frac{(\alpha-1) x_{i}^{2} e^{-x_{i} / \beta}}{\beta^{3}\left[1-\left(1+\frac{x_{i}}{\beta}\right) e^{-x_{i} / \beta}\right]}=0 .
$$

It follows, by the asymptotic properties of the $M L E$, that

$$
\hat{\beta}_{M L E S H K} \approx N\left(\beta, \frac{1}{n \phi_{22}}\right),
$$

where $\phi_{22}$ is the single information about $\beta$ which is defined in (5.6).

\subsection{Approximate confidence intervals}

Since the MLEs of the unknown parameters $\alpha$ and $\beta$ cannot be derived in closed form, it is not easy to derive the exact distributions of the $M L E s$. Hence, we cannot obtain exact confidence intervals for the parameters. We must use the large sample approximation. It is known that the asymptotic distribution of the $M L E$ is $\left[\sqrt{n}\left(\hat{\alpha}_{M L E}-\alpha\right), \sqrt{n}\left(\hat{\beta}_{M L E}-\beta\right)\right] \rightarrow N_{2}\left(0, I^{-1}(\alpha, \beta)\right)$, we can refer Lawless [23], where $I^{-1}(\alpha, \beta)$, the inverse of observed information matrix of the unknown parameters $\Theta=(\alpha, \beta)$, is

$$
\begin{aligned}
I^{-1}(\Theta) & =\left(\begin{array}{cc}
-\frac{\partial^{2} l(\alpha, \beta)}{\partial^{2} \alpha} & -\frac{\partial^{2} l(\alpha, \beta)}{\partial \alpha \partial \beta} \\
-\frac{\partial^{2} l(\alpha, \beta)}{\partial \alpha \partial \beta} & -\frac{\partial^{2} l(\alpha, \beta)}{\partial^{2} \beta}
\end{array}\right)_{(\alpha, \beta)=(\hat{\alpha}, \hat{\beta})}^{-1} \\
& =\left(\begin{array}{cc}
\operatorname{Var}(\hat{\alpha}) & \operatorname{Cov}(\hat{\alpha}, \hat{\beta}) \\
\operatorname{Cov}(\hat{\alpha}, \hat{\beta}) & \operatorname{Var}(\hat{\alpha})
\end{array}\right) .
\end{aligned}
$$

The derivatives in $I(\Theta)$ are given in

$$
\begin{gathered}
\frac{\partial^{2} l(\alpha, \beta \mid x)}{\partial \alpha^{2}}=-\frac{n}{\alpha^{2}} . \\
\frac{\partial^{2} l(\alpha, \beta \mid x)}{\partial \alpha \partial \beta}=\sum_{i=1}^{n} \frac{(\alpha-1) x^{2} e^{-x_{i} / \beta}}{\beta^{4}\left[1-\left(1+\frac{x_{i}}{\beta}\right) e^{-x_{i} / \beta}\right]}=\frac{\partial^{2} l(\alpha, \beta \mid x)}{\partial \beta \partial \alpha} . \\
\frac{\partial^{2} l(\alpha, \beta \mid x)}{\partial \beta^{2}}=\frac{2 n}{\beta^{2}}-\frac{2}{\beta^{3}} \sum_{i=1}^{n} x_{i}+\sum_{i=1}^{n} \frac{3(\alpha-1) x_{i}^{2} e^{-x_{i} / \beta}}{\beta^{4}\left[1-\left(1+\frac{x_{i}}{\beta}\right) e^{-x_{i} / \beta}\right]}
\end{gathered}
$$




$$
-\sum_{i=1}^{n} \frac{(\alpha-1) x_{i}^{3} e^{-x_{i} / \beta}}{\beta^{5}\left[1-\left(1+\frac{x_{i}}{\beta}\right) e^{-x_{i} / \beta}\right]}+\sum_{i=1}^{n} \frac{(\alpha-1) x_{i}^{3} e^{-2 x_{i} / \beta}\left[1-\left(1+\frac{x_{i}}{\beta}\right)\right]}{\beta^{5}\left[1-\left(1+\frac{x_{i}}{\beta}\right) e^{-x_{i} / \beta}\right]^{2}} .
$$

The above approach is used to derive approximate $100(1-\tau) \%$ confidence intervals of the parameters $\alpha$ and $\beta$ of the forms

$$
\hat{\alpha} \pm z_{\tau / 2} \sqrt{\operatorname{var}(\hat{\alpha})}
$$

and

$$
\hat{\beta} \pm z_{\tau / 2} \sqrt{\operatorname{var}(\hat{\beta})}
$$

where $z_{\tau / 2}$ is the upper $(\tau / 2)$ th percentile of the standard normal distribution.

\section{Bayesian estimation}

In this section we consider Bayesian inference of the unknown parameters of the EMED. It is assumed that $\alpha$ and $\beta$ has the independent gamma prior distributions with probability density functions

$$
h(\alpha) \propto \alpha^{a-1} e^{-b \alpha}, \quad \alpha>0 .
$$

and

$$
h(\beta) \propto \beta^{c-1} e^{-d \beta}, \beta>0 .
$$

The hyper-parameters $a, b, c$, and $d$ are known and non-negative. If both the parameters $\alpha$ and $\beta$ are unknown, joint conjugate priors do not exist. It is not unreasonable to assume independent gamma priors on the shape and scale parameters for a two-parameter $E M E D$, because gamma distributions are very flexible, and the Jeffreys (non-informative) prior, introduced by Jeffreys [15] is a special case of this. The joint prior distribution in this case is

$$
h(\alpha, \beta) \propto \alpha^{a-1} e^{-b \alpha} \beta^{c-1} e^{-d \beta}, \alpha, \beta>0 .
$$

Combining (6.2) with (7.3) and using Bayes theorem, the joint posterior distribution is derived as

$$
\pi(\alpha, \beta \mid x)=\alpha^{n+a-1} \beta^{c-2 n-1} e^{-b \alpha-d \beta} \frac{1}{I_{0}} \prod_{i=1}^{n} \frac{x_{i} e^{-x_{i} / \beta}}{\left[1-\left(1+\frac{x_{i}}{\beta}\right) e^{-x_{i} / \beta}\right]^{\alpha-1}} .
$$

where

$$
I_{0}=\int_{0}^{\infty} \int_{0}^{\infty} \alpha^{n+a-1} \beta^{c-2 n-1} \prod_{i=1}^{n} \frac{x_{i} e^{-x_{i} / \beta} e^{-b \alpha-d \beta}}{\left[1-\left(1+\frac{x_{i}}{\beta}\right) e^{-x_{i} / \beta}\right]^{\alpha-1}} d \alpha d \beta .
$$

The marginal posterior distribution of a parameter is obtained by integrating the joint posterior distribution with respect to the other parameter. Hence, the marginal posterior probability density functions of $\alpha$ and $\beta$ are given, respectively, by

$$
\pi_{1}(\alpha \mid x)=\frac{\alpha^{n+a-1} e^{-b \alpha}}{I_{0}} \int_{0}^{\infty} \beta^{c-2 n-1} \prod_{i=1}^{n} \frac{x_{i} e^{-x_{i} / \beta} e^{-d \beta}}{\left[1-\left(1+\frac{x_{i}}{\beta}\right) e^{-x_{i} / \beta}\right]^{\alpha-1}} d \beta
$$

and

$$
\pi_{2}(\beta \mid x)=\frac{\beta^{c-2 n-1} e^{-d \beta}}{I_{0}} \int_{0}^{\infty} \alpha^{n+a-1} \prod_{i=1}^{n} \frac{x_{i} e^{-x_{i} / \beta} e^{-b \alpha}}{\left[1-\left(1+\frac{x_{i}}{\beta}\right) e^{-x_{i} / \beta}\right]^{\alpha-1}} d \alpha .
$$

Next, we must consider the question of what loss function will be used to derive the estimators from the marginal posterior distributions. 


\subsection{Bayes estimators under the general entropy loss function}

Calabria and Pulcini [7] have derived the point estimation under asymmetric loss function from left-truncated exponential samples. According to this theory, the Bayes estimators for the parameters $\alpha$ and $\beta$ for the probability density function (1.4) under the general entropy loss function may be defined as

$$
\hat{\alpha}_{B G E}=\left[E(\alpha)^{-q}\right]^{-1 / q}
$$

and

$$
\hat{\beta}_{B G E}=\left[E(\beta)^{-q}\right]^{-1 / q}
$$

respectively, provided that $E(\alpha)^{-q}$ and $E(\beta)^{-q}$ exist and are finite. These estimators can be expressed as

$$
\hat{\alpha}_{B G E}=\left[\frac{I_{\alpha}}{I_{0}}\right]^{-1 / q}
$$

and

$$
\hat{\beta}_{B G E}=\left[\frac{I_{\beta}}{I_{0}}\right]^{-1 / q},
$$

where

$$
I_{\alpha}=\int_{0}^{\infty} \int_{0}^{\infty} \alpha^{n+a-q-1} \beta^{c-2 n-1} \prod_{i=1}^{n} \frac{x_{i} e^{-x_{i} / \beta} e^{-b \alpha-d \beta}}{\left[1-\left(1+\frac{x_{i}}{\beta}\right) e^{-x_{i} / \beta}\right]^{\alpha-1}} d \alpha d \beta
$$

and

$$
I_{\beta}=\int_{0}^{\infty} \int_{0}^{\infty} \alpha^{n+a-1} \beta^{c-q-2 n-1} \prod_{i=1}^{n} \frac{x_{i} e^{-x_{i} / \beta} e^{-b \alpha-d \beta}}{\left[1-\left(1+\frac{x_{i}}{\beta}\right) e^{-x_{i} / \beta}\right]^{\alpha-1}} d \alpha d \beta
$$

All the double integrals above have no closed form. Therefore, we will implement the Metropolis-Hastings (M-H) algorithm to compute the estimators. The $\mathrm{M}-\mathrm{H}$ algorithm is a powerful Markov Chain Monte Carlo algorithm. The M-H algorithm was introduced by Metropolis et al. [27]. For a discussion of the algorithm, the reader is referred to any Bayesian statistics textbook. In this chapter, we consider three special cases of the general entropy loss function, corresponding to $q=-1, q=1$ and $q=-2$. It should be mentioned that for $q=-1$ the general entropy loss function simplifies to the squared-error loss function. The weighted squared-error loss function results from $q=1$. For $q=-2$, the general entropy loss function is referred to as the precautionary loss function which is an asymmetric loss function.

\subsection{Two-sided Bayes probability intervals}

The Bayesian method to interval estimation is much more direct than the frequentist method based on confidence intervals. Once the marginal posterior distribution of $\alpha$ has been obtained, a symmetric $100(1-\tau) \%$ two-sided Bayes probability interval estimate of $\alpha$, denoted by $\left[\alpha_{L}, \alpha_{U}\right]$, can be obtained by solving the two equations

$$
\int_{0}^{\alpha_{L}} \pi_{1}(w \mid x) d w=\frac{\tau}{2}
$$

and

$$
\int_{\alpha_{U}}^{\infty} \pi_{1}(w \mid x) d w=\frac{\tau}{2}
$$

for the limits $\alpha_{L}$ and $\alpha_{u}$. Similarly, a symmetric $100(1-\tau) \%$ two-sided Bayes probability interval estimate of $\beta$, denoted by $\left[\beta_{L}, \beta_{U}\right]$, can be obtained by solving

$$
\int_{0}^{\beta_{L}} \pi_{2}(w \mid x) d w=\frac{\tau}{2}
$$

and

$$
\int_{\beta_{U}}^{\infty} \pi_{2}(w \mid x) d w=\frac{\tau}{2}
$$

for the limits $\beta_{L}$ and $\beta_{U}$. Again, these equations cannot be solved in close form. 


\section{Empirical Bayes estimation}

In the preceding sections, we have assumed the hyper-parameters $a, b, c$, and $d$ are known. Empirical Bayes estimation addresses the question of estimating the hyper-parameters from existing data. When the current sample is observed, assume that $p$ past samples $X_{j 1}, X_{j 2}, \ldots, X_{j n}$, for $j=1,2, \ldots, p$, are available. Each sample is assumed to be sample of size $n$ from a $E M E$ distribution. The likelihood function for each sample $j$ is given by

$$
L(\alpha, \beta ; x)=\frac{\alpha^{n}}{\beta^{2 n}} \prod_{i=1}^{n}\left[1-\left(1+\frac{x_{j i}}{\beta}\right) e^{-x_{j i} / \beta}\right]^{\alpha-1} x_{j i} e^{-x_{j i} / \beta} .
$$

For each sample $j$, let $\hat{\alpha}_{j}$ and $\hat{\beta}_{j}$ be the maximum likelihood estimates for $\alpha$ and $\beta$, respectively, based on sample $j$, which are obtained from (8.1). We then calculate the mean and variance of the maximum likelihood estimators for each of the $j$ samples, equate these to the mean and variance of the gamma prior distribution, and solve for the hyper-parameters. We can find $\hat{a}$ and $\hat{b}$, estimators for $a$ and $b$, by solving

$$
\frac{1}{p} \sum_{i=1}^{p} \hat{\alpha}_{j}=\frac{b}{a}
$$

and

$$
\frac{1}{(p-1)} \sum_{i=1}^{p}\left(\hat{\alpha}_{j}-\frac{1}{p} \sum_{i=1}^{p} \hat{\alpha}_{j}\right)^{2}=\frac{b}{a^{2}} .
$$

We can find $\hat{c}$ and $\hat{d}$, estimators for $c$ and $d$, by solving

$$
\frac{1}{p} \sum_{i=1}^{p} \hat{\beta}_{j}=\frac{d}{c}
$$

and

$$
\frac{1}{(p-1)} \sum_{i=1}^{p}\left(\hat{\beta}_{j}-\frac{1}{p} \sum_{i=1}^{p} \hat{\beta}_{j}\right)^{2}=\frac{d}{c^{2}} .
$$

Solving the above equations yields the estimators for the hyper-parameters

$$
\hat{a}=\frac{\left(\frac{1}{p} \sum_{i=1}^{p} \hat{\alpha}_{j}\right)}{\left(\frac{1}{p-1} \sum_{i=1}^{p}\left(\hat{\alpha}_{j}-\frac{1}{p} \sum_{i=1}^{p} \hat{\alpha}_{j}\right)^{2}\right)}
$$

and

$$
\hat{b}=\frac{\left(\frac{1}{p} \sum_{i=1}^{p} \hat{\alpha}_{j}\right)^{2}}{\left(\frac{1}{p-1} \sum_{i=1}^{p}\left(\hat{\alpha}_{j}-\frac{1}{p} \sum_{i=1}^{p} \hat{\alpha}_{j}\right)^{2}\right)}
$$

for the prior distribution for $\alpha$. Similarly, estimators for the hyper-parameters for the prior distribution for $\beta$ can be found as

$$
\hat{c}=\frac{\left(\frac{1}{p} \sum_{i=1}^{p} \hat{\beta}_{j}\right)}{\left(\frac{1}{p-1} \sum_{i=1}^{p}\left(\hat{\beta}_{j}-\frac{1}{p} \sum_{i=1}^{p} \hat{\beta}_{j}\right)^{2}\right)}
$$

and

$$
\hat{d}=\frac{\left(\frac{1}{p} \sum_{i=1}^{p} \hat{\beta}_{j}\right)^{2}}{\left(\frac{1}{p-1} \sum_{i=1}^{p}\left(\hat{\beta}_{j}-\frac{1}{p} \sum_{i=1}^{p} \hat{\beta}_{j}\right)^{2}\right)} .
$$

The empirical Bayes estimators of $\alpha$ and $\beta$ are found by substituting $\hat{a}, \hat{b}, \hat{c}$, and $\hat{d}$ into (7.6) and (7.7) and proceeding as before. 


\section{Numerical Experiments and Discussions}

In this section, we present the results of some numerical experiment to examine and compare the performance of maximum likelihood and Bayes estimators for the two-parameter $E M E D$ proposed in the previous section. We apply Monte Carlo simulations to examine the performance of the $M L E s$, mainly with respect to their biases and mean squared errors $(M S E s)$ for different sizes and different parameter values, since $\beta$ is the scale parameter and all the estimaters are scale invariant, we take $\beta=1$ in all computations we set $\alpha=0.25,0.5,1.0,2,2.5$ and $n=10,20,50,100$. We compute the biase estimates and $M S E s$ over 1000 replications for different cases. Table 1 and Table 2 lists the bias estimates of maximum likelihood estimators of the two model parameters along with the respective $M S E s$. From the results, we see that as the sample size $n$ increases, $M S E s$ of the parameters are decreases. It indicate that the deduce asymptotically unbiased and consistent estimators of the parameters $\alpha$ and $\beta$.

The Bayes estimators cannot be found in closed form. Therefore, we use the Metropolis-Hastings algorithm to compute Bayes estimates. We use informative priors for both $\alpha$ and $\beta$. The chosen hyper-parameters are $a=c=4$ and $b=d=1$. Bayes estimators are computed using the general entropy loss function with $q=-2,-1,1$. This allows us to consider the Bayes estimators under both symmetric and asymmetric loss functions. The proposal distribution used for the $\mathrm{M}-\mathrm{H}$ algorithm is a chi-square distribution. We generate 5000 samples after 5000 burn-in samples. To evaluate the convergence of the $\mathrm{M}-\mathrm{H}$ algorithm, we use Gelman-Rubin diagnostics, using the R package coda. For both $\alpha$ and $\beta$, three different Markov chains are simulated. The potential scale reduction factor (psrf) for $\alpha$ is 1.01, and the $\operatorname{psr} f$ for $\beta$ is 1 . Since both are close to 1 , we can conclude that the Markov chains converged quite well to the stationary distribution.

The following observations can be drawn from the Tables 1,2 , and 3

1. All the estimators show the property of consistency i.e., the bias and MSE decreases as sample size increases.

2. The bias of $\hat{\alpha}$ generally increases with increasing $\alpha$ for any $n$ and for all methods of estimation.

3. The bias of $\hat{\beta}$ generally increases with increasing $\beta$ for any given $n$ and for all methods of estimation.

4. All estimates of $\alpha$ and $\beta$ are biased and their bias distribution have different spreads. On the other hand, the distributions of MSE seem to have fairly similar spreads.

5. We observe that the Bayesian estimates based on WSB are considerably less biased than the corresponding Bayesian estimates based on SB and PL.

6. We also observe that the Bayesian estimates based on WSB have a much smaller MSE than the corresponding Bayesian estimates based on their counterparts in all cases considered.

7. Results show that the performance of the Bayes estimates is better than the MLEs, in terms of the bias and MSE.

8. Finally, this clearly demonstrates the efficiency of inference based on WSB and also the usefulness of the Bayesian estimates based on WSB developed here for the parameters of the exponentiated moment exponential distribution.

Table 1. Average bias estimates of $\alpha$ are presented, when $\beta$ is known and their associated MSEs (in parentheses)

\begin{tabular}{ccrccc}
\hline$n$ & $\alpha=0.25$ & $\alpha=0.5$ & $\alpha=1.0$ & $\alpha=2.0$ & $\alpha=2.5$ \\
\hline 10 & 0.12179 & 0.13471 & 0.14358 & 0.18824 & 0.23975 \\
& $(0.03759)$ & $(0.16337)$ & $(0.16887)$ & $(0.67852)$ & $(0.98213)$ \\
20 & 0.03697 & 0.03568 & 0.05673 & 0.08734 & 0.17528 \\
& $(0.02178)$ & $(0.04824)$ & $(0.07152)$ & $(0.08953)$ & $(0.17032)$ \\
50 & 0.02926 & 0.03272 & 0.03813 & 0.03972 & 0.05792 \\
& $(0.00126)$ & $(0.00631)$ & $(0.02561)$ & $(0.09573)$ & $(0.11958)$ \\
100 & 0.02872 & 0.03156 & 0.03507 & 0.03603 & 0.05579 \\
& $(0.00119)$ & $(0.00203)$ & $(0.01203)$ & $(0.03527)$ & $(0.05917)$ \\
\hline
\end{tabular}

Table 4-5 lists some numerical values for the first four moments, variances, skewness and kurtosis of order statistics and lower record values. The parameter values are taken as $\alpha=2$ and $\beta=5$. 
Table 2. Average bias estimates of $\beta$ are presented, when $\alpha$ is known and their associated MSEs (in parentheses)

\begin{tabular}{ccrccc}
\hline$n$ & $\alpha=0.25$ & $\alpha=0.5$ & $\alpha=1.0$ & $\alpha=2.0$ & $\alpha=2.5$ \\
\hline 10 & 0.19287 & 0.19868 & 0.22039 & 0.38992 & 0.42352 \\
& $(0.60359)$ & $(0.27659)$ & $(0.08038)$ & $(0.03731)$ & $(0.04178)$ \\
20 & 0.07357 & 0.09962 & 0.13274 & 0.18203 & 0.22972 \\
& $(0.23078)$ & $(0.08351)$ & $(0.02171)$ & $(0.02756)$ & $(0.01763)$ \\
50 & 0.02702 & 0.03979 & 0.04692 & 0.05099 & 0.05371 \\
& $(0.06926)$ & $(0.03752)$ & $(0.01078)$ & $(0.00572)$ & $(0.00572)$ \\
100 & 0.02207 & 0.02976 & 0.039832 & 0.04875 & 0.04903 \\
& $(0.05872)$ & $(0.00181)$ & $(0.00581)$ & $(0.00690)$ & $(0.00792)$ \\
\hline
\end{tabular}

Table 3. Average bias and $M S E s$ (in parentheses) of empirical Bayes estimators for $\alpha$ and $\beta$

\begin{tabular}{ccrcccc}
\hline$n$ & $\hat{\alpha}_{S B}$ & \multicolumn{1}{c}{$\hat{\alpha}_{W S B}$} & $\hat{\alpha}_{P L}$ & $\hat{\beta}_{S B}$ & $\hat{\beta}_{W S B}$ & $\hat{\beta}_{P L}$ \\
\hline 10 & 0.8062 & 0.2078 & 0.4984 & 1.3437 & 0.6934 & 0.6524 \\
& $(0.5807)$ & $(0.2143)$ & $(0.3065)$ & $(1.6707)$ & $(0.6389)$ & $(6.4378)$ \\
20 & 0.6254 & 0.1801 & 0.3267 & 0.4734 & 0.4068 & 0.4502 \\
& $(0.0894)$ & $(0.0254)$ & $(0.0302)$ & $(0.3724)$ & $(0.0804)$ & $(0.9361)$ \\
50 & 0.4262 & 0.1504 & 0.1943 & 0.3264 & 0.3032 & 0.3406 \\
& $(0.0307)$ & $(0.0191)$ & $(0.0014)$ & $(0.4932)$ & $(0.0462)$ & $(0.7402)$ \\
100 & 0.3213 & 0.0385 & 0.1567 & 0.2045 & 0.2871 & 0.1841 \\
& $(0.0189)$ & $(0.0021)$ & $(0.0046)$ & $(0.2186)$ & $(0.03482)$ & $(0.4847)$ \\
\hline
\end{tabular}

Note: The subscript WSB stands for the Bayes estimate under weighted squared error loss function; SB represent the squared error loss function, PL stands for the precautionary loss function.

Table 4. First four moments, variances, skewness and kurtosis of some order statistics

\begin{tabular}{cccccccc}
\hline$X_{r: 5} \downarrow$ & $j=1$ & $\mathrm{j}=2$ & $j=3$ & $j=4$ & Variance & Skewness & Kurtosis \\
\hline 1 & 0.231304 & 0.107003 & 0.074250 & 0.068697 & 0.053501 & 3.187607 & 8.999998 \\
2 & 0.346955 & 0.187255 & 0.139219 & 0.133101 & 0.066877 & 5.762311 & 7.080104 \\
3 & 0.424056 & 0.252645 & 0.197657 & 0.194060 & 0.072822 & 2.141937 & 6.480995 \\
4 & 0.481882 & 0.308376 & 0.251153 & 0.252152 & 0.076166 & 12.42950 & 6.193655 \\
5 & 0.528143 & 0.357240 & 0.300732 & 0.307800 & 0.078305 & 16.28332 & 6.025762 \\
\hline
\end{tabular}

Table 5. First four moments, variances, skewness and kurtosis of some lower record values

\begin{tabular}{cccccccc}
\hline$r \downarrow$ & $j=1$ & $\mathrm{j}=2$ & $j=3$ & $j=4$ & Variance & Skewness & Kurtosis \\
\hline 1 & 4.323324 & 37.38225 & 484.8467 & 8384.597 & 18.69112 & 4.000005 & 9.000004 \\
2 & 8.646647 & 112.14676 & 1939.3869 & 41922.99 & 37.38226 & 1.999998 & 6.000002 \\
3 & 12.969971 & 224.2935 & 4848.467 & 125768.96 & 56.07335 & 1.333339 & 5.000002 \\
4 & 17.29329 & 373.8225 & 9696.935 & 293460.91 & 74.76462 & 0.999993 & 4.499990 \\
5 & 21.61662 & 560.7338 & 16969.636 & 586921.8 & 93.45554 & 0.800008 & 4.199993 \\
\hline
\end{tabular}

\section{Applications}

The recurrence relations obtained in the preceding sections allow us to evaluate the means, variances and covariances of all order statistics for all sample sizes in a simple recursive manner. Means, variances and covariances of all order statistics can be used for various inferential purposes; for example, they are useful in determining BLUEs of location/scale parameters and best linear unbiased predictors (BLUPs) of censored failure times. More details on BLUEs and BLUPs based on order statistics can be seen in Balakrishnan and Cohen [3] and Arnold et al. [2].

\section{Concluding remarks}

The EMED provided excellent model for life time data for a variety of situations. Thus, it is important for the analyst to have reliable statistical methods to use for this distribution. We have provided in this study, a new explicit expressions and recurrence relations for single and product moments of $l g o s$ from the EMED. Further, a characterizing result of this distribution on using the recurrence relation of single moments of the lgos discussed. We have examined and compared the different methods, including Bayesian methods under different loss function. 


\section{Acknowledgements}

The author expresses their sincere thanks to the editor and anonymous reviewers for their useful comments on an earlier version of this manuscript which led to this improved version.

\section{References}

[1] Ahsanullah, M., On lower generalized order statistics and a characterization of power function distribution. Stat. Methods, 7 (2005), 16-28.

[2] Arnold, B. C., Balakrishnan, N. and Nagaraja, H. N., A First Course in Order Statistics. John Wiley, New York, (1992).

[3] Balakrishnan, N. and Cohan, A.C., Order Statistics and Inference: Estimation Methods. Academic Press, San Diego, (1991).

[4] Brown, M., Low Density Traffic Streams. Advances in Applied Probability, 4 (1972), 177-192.

[5] Bieniek, M. and Szynal, D., Characterizations of distributions via linearity of regression of generalized order statistics. Metrika, 58 (2003), 259-271.

[6] Burkschat, M., Cramer, E. and Kamps, U., Dual generalized order statistics. Metron, LXI (2003), 13-26.

[7] Calabria, R. and Pulcini, G., Point estimation under asymmetric loss functions for left-truncated exponential samples. Comm. Statist. Theory Methods, 25 (1996), 585-600.

[8] Chandler, K. N., The distribution and frequency of record values, J. Roy. Stat. Soc. B. 14 (1952), 220-228.

[9] Cramer, E., Kamps, U. and Keseling, C., Characterization via linear regression of ordered random variables: a unifying approach. Communications in Statistics-Theory and Methods, 33 (2004), 2885-2911.

[10] Dara, S.T. and Ahmad, M., Recent Advances in Moment Distributions and their Hazard Rate. Ph.D. Thesis. National College of Business Administration and Economics, Lahore, Pakistan, (2012).

[11] Gupta, R.D. and Kundu, D.,Generalized exponential distributions; An alternative to gamma or Weibull distribution. Biometrical Journal, 43(2001), 117-130.

[12] Gupta, R. D. and Kundu, D., On the comparison of Fisher information of the Weibull and generalized exponential distributions, Journal of Statistical Planning and Inference, 136 (2006), 3130-3144.

[13] Gy, P., Sampling of Particulate Material: Theory and Practice. Elsevier: Amsterdam, 431 (1982).

[14] Hasnain, S. A., Exponentiated moment Exponential Distribution. Ph.D. Thesis. National College of Business Administration and Economics, Lahore, Pakistan, (2013).

[15] Jeffreys, H., An invariant form for the prior probability in estimation problems, Proceedings of Royal Society of London. Series A, Mathematical and Physical Sciences 186(1946), 435-461.

[16] Kamps, U., A concept of generalized order statistics. B.G. Teubner Stuttgart, (1995).

[17] Keseling, C., Conditional distributions of generalized order statistics and some characterizations. Metrika, 49 (1999), 27-40.

[18] Khan, R.U. and Kumar, D., On moments of generalized order statistics from exponentiated Pareto distribution and its characterization. Appl. Math. Sci. (Ruse), 4 (2010), 2711-2722.

[19] Krumbein, W.C. and Pettijohn, F. J. Manual of sedimentary petrography. Appleton Century-Crofts, Inc., New York, 230-233, (1938).

[20] Kumar, D., Relations for moments of lower generalized order statistics from exponentiated inverted Weibull distribution. Tamsui Oxford journal of Mathematical Science. 30 (2014), 1-21. 
[21] Kumar, D., Exponentiated Gamma Distribution Based On Ordered Random Variables. Applied Mathematics and E-Notes, 15 (2015), 105-120.

[22] Kumar, D., Lower Generalized Order Statistics Based On Inverse Burr Distribution, American Journal of Mathematical and Management Sciences, 35 (2015), 15-35.

[23] Lawless, J. F., Statistical models and methods for lifetime data, 2nd Edition, Wiley, New York, (1982).

[24] Lin, G. D., On a moment problem. Tohoku Math. Journal, 38 (1986), 595-598.

[25] Martz, H. F. and Waller, R. A., Bayesian Reliability Analysis, Wiley, New York, (1982).

[26] Mbah, A.K. and Ahsanullah, M., Some characterization of the power function distribution based on lower generalized order statistics. Pakistan J. Statist., 23(2007), 139-146.

[27] Metropolis, N., Rosenbluth, A. W., Rosebluth, M. N., Teller, A. H. and Teller, E., Equations of state calculations by fast computing machines, Journal of Chemical Physics, 21 (1953), 1087-1092.

[28] Pawlas, P. and Szynal, D., Recurrence relations for single and product moments of lower generalized order statistics from the inverse Weibull distribution. Demonstratio Math., XXXIV (2001), 353-358.

[29] Preston, F.W., The canonical distribution of commonness and rarity, Ecology 43 (1962), 186-215.

[30] Taillie, C., Patil, G.P. and Hennemuth, R.,Modelling and analysis of recruitment distributions. Ecological and Environmental Statistics, 2 (1995), 315-329.

[31] Temkin, N., Interactive information and distributional length biased survival models. Unpublished Ph.D. dissertation, University of New York at Buffalo, (1976).

[32] Warren, W., Statistical distributions in forestry and forest products research. In: Patil, G.P, Kotz, S. and Ord, J.K. (eds) Statistical distributions in scientific work, Vol. 2, D. Reidel, Dordrecht, The Netherlands, 360-384, (1975).

[33] Zelen, M., Problems in cell kinetics and the early detection of disease, in Reliability and Biometry, F. Proschan \& R.J. Serfling, eds, SIAM, Philadelphia, 701-706, (1974).

[34] Zelen M., Theory of early detection of breast cancer in the general population, Breast Cancer: Trends in Research and Treatment (ed. J.C. Heuson, W.H. Mattheiem, M. Rozenweig), Raven Press, New York, 287-301, (1976).

[35] Zheng, G., On the Fisher information matrix in type II censored data from the exponentiated exponential family, Biometrical Journal, 44(2002), 353-357.

\section{Affiliations}

DEVENDRA KUMAR

AdDRESS: Department of Statistics, Amity Institute of Applied Amity University Noida.

E-MAIL: devendrastats@gmail.com 